\title{
La seconda metà del XVI secolo: un punto di svolta nell'organizzazione difensiva del Regno di Sardegna
}

The second half of the sixteenth century: a turning point in the organization of the Kingdom of Sardinia defense

\section{Giovanni Serreli}

Istituto di Storia dell'Europa Mediterranea - Consiglio Nazionale delle Ricerche, Cagliari, Italy, serreli@isem.cnr.it

\begin{abstract}
In the second half of the sixteenth century, in accordance with the centralization and reorganization of the administrative and military apparatus desired by Philip II in the States of the Iberian Monarchy, military defense was reorganized in the Kingdom of Sardinia, against the threatened Franc-Turkish invasion of the Island. Until that time, in fact, the defense of the Kingdom was based only on the modernization of the main Royal Cities fortifications. The inspiring principle of this strategy was the medieval one: to defend the Kingdom, it would have been enough to defend the Royal Cities of Cagliari and Alghero. Instead, starting from the Parliament wanted in 1558 by Philip II and summoned by the viceroy Alvaro de Madrigal, attention is also paid to the defense of the coastal perimeter of the whole island. In this Parliament, convened with the sole purpose of providing for the defense of the Kingdom against the looming Franc-Turkish threat, the foundations are laid for the reorganization of the administrative apparatus of the Kingdom, limiting the centrifugal tendencies of local feudalism; but above all, they are laid the foundations for the creation of a homogeneous defense system, abandoning the extemporaneous initiatives taken up to that time. By the end of the century, reports for the construction of a static defense system will be commissioned and the Royal Administration of Towers will be established, with the task of building, arming and maintaining the static defense system.
\end{abstract}

Keywords: Philip II, Kingdom of Sardinia, fortifications, military defense system.

Il Regno di Sardegna, pur essendo periferico nel contesto della vasta Monarchia Iberica (Fig. 1), mantenne una evidente importanza strategia durante tutto il XVI secolo a causa del contesto geopolitico Mediterraneo; i vari fronti bellici aperti da Carlo V sia nel nord Europa che nel Mediterraneo occidentale, nel momento di massimo pericolo nel confronto con l'Impero Ottomano alleato dei francesi, rendevano il Regno sardo un baluardo fondamentale per la sicurezza degli Stati mediterranei della Monarchia (Mattone, 2007, pp. 475-498). Proprio a cavaliere della metà del Cinquecento, l'Isola si trovava tra due fuochi: da un lato la pressione turca sul Mediterraneo e la guerra di corsa delle reggenze barbaresche, dall'altro il pericolo che la Corsica, e successivamente la Sardegna stessa cadessero in mano francese. Il rischio di perdere questo strategico avamposto pareva imminente ed attuale (Mele, 2015, pp. 135-146; Mattone, 2003, pp. 153-160, 177-179) ed era recentissimo il saccheggio e la distruzione di Terranova (Olbia) da parte della flotta turca, che aveva evidenziato l'estrema fragilità difensiva del Regno sardo (Serreli, 2007, pp. 175-185). 


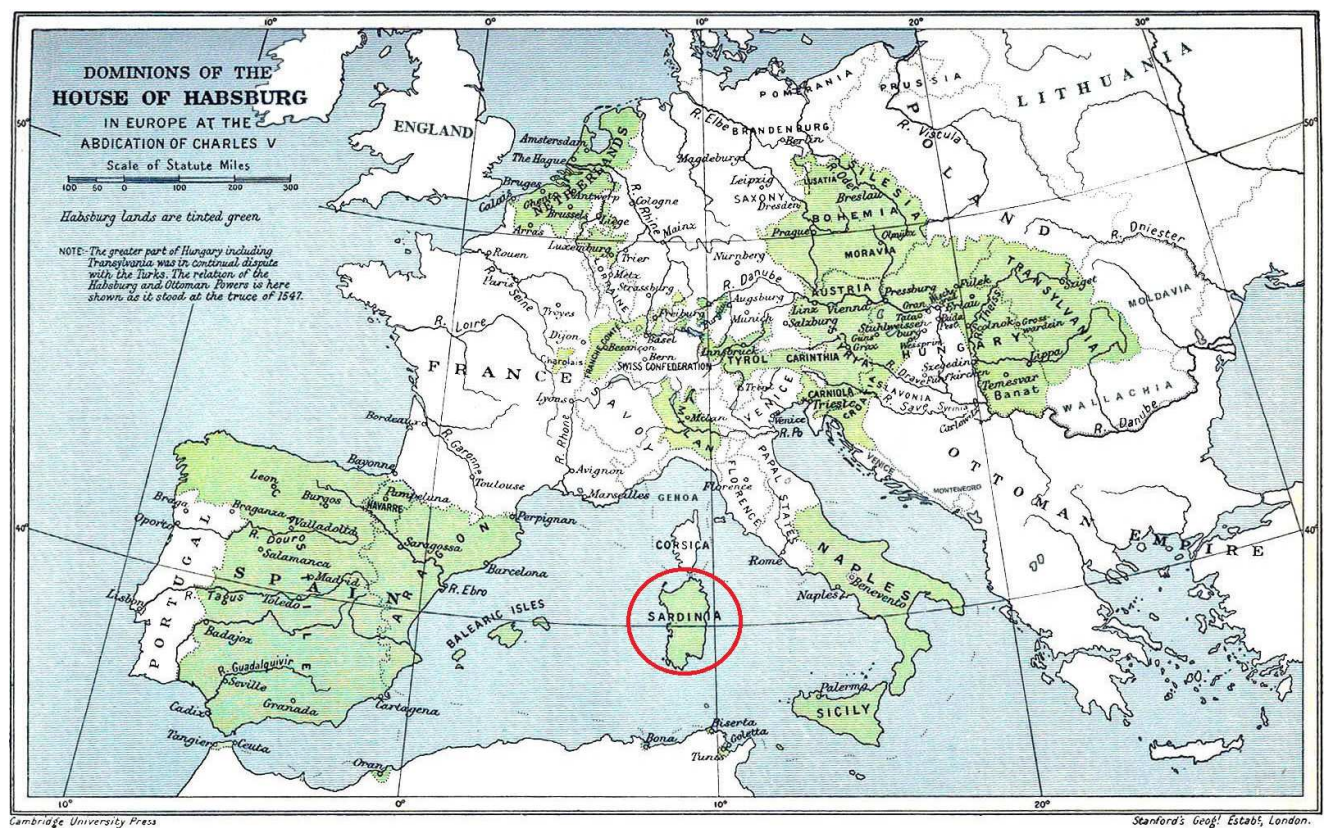

Fig. 1. La Monarchia Iberica nel The Cambridge Modern History Atlas (1912).

Perciò, quando nel gennaio del 1556 il principe Filippo divenne sovrano della Corona d'Aragona (Buyreu, 1999, p. 342; Spagnoletti, 2018), era cosciente della improcrastinabile necessità di provvedere alla difesa dei suoi Stati mediterranei (Cancila, 2007; Anatra, Mele, Murgia, Serreli, 2008) e, fra questi del Regno di Sardegna (Anatra, 1984, pp. 436-511; Murgia, 2012, pp. 61119; Mele, 2017), anche se, sulla base di paradigmi ereditati dai secoli precedenti e in linea con una strategia prettamente difensiva che risentiva ancora delle istruzioni date a Filippo da suo padre, l'imperatore Carlo (Cipollone, 2012, pp. 36-37).

Per tutti questi motivi, i primi due più importanti provvedimenti del sovrano per il Regno di Sardegna riguardarono la nomina di un nuovo luogotenente e la convocazione di un Parlamento straordinario.

In primo luogo, a pochi mesi dalla sua ascesa al trono, il 26 agosto 1556 Filippo II nominò il suo primo luogotenente nel Regno di Sardegna, il militare Alvaro de Madrigal; nelle istruzioni inviategli dalla principessa Giovanna sono poste in evidenza quelle inerenti la difesa e le fortifica- zioni delle Città, già avviate e da ultimare con estrema urgenza, considerato il contesto geopolitico sopra descritto; l'architetto Rocco Capellino si trovava a Cagliari (Fig. 3) già da alcuni anni (Nocco, 2009, pp. 137-139). Inoltre, veniva raccomandato al nuovo viceré di mantenere stretti contatti con i luogotenenti di Sicilia e Napoli, con il capitano de La Goletta e con gli ambasciatori a Roma e a Genova per essere informato costantemente dei movimenti del nemico e aggiornare periodicamente la corte (Buyreu, 2000, pp. 151-161). Anche attraverso la nomina del Madrigal e le istruzioni date, siamo quindi in grado di leggere i primi passi del disegno del re Prudente per la riorganizzazione delle difese statiche del Regno di Sardegna parallelamente a quelle degli altri Stati della Corona. Del resto il Madrigal -oltre a essere legato ai Cardona, una delle famiglie più vicine alla causa imperiale e alla sua guerra su vari fronti per garantire la realizzazione del sogno di «un solo re, un solo impero, e una sola spada» (Elliot, 2002, p. 285)- è un fedele militare che ha dato prova del suo valore in vari campi di battaglia (Manconi, 2010, pp. 200-201). A conferma dell'attenzione che ebbe in campo militare, qui sottolineiamo che 
egli fu il primo viceré a convocare un Consiglio di Guerra, distinto dal Consiglio Regio (Oliva, 2005, pp. 205-238), di cui facevano parte i personaggi con maggiore esperienza militare e nella difesa: "Et die veneris intitulato 21 dicti mensis junii, ad celebrationem dicti presentis Regii Generalis Parlamenti [...] per la deliberatio presa en lo Consell de Guerra y en altres necessitats concorrent per a la defensio del present Regne [...]" (Archivio di Stato di Cagliari [ASC], Antico Archivio Regio [AAR], Parlamenti, vol. 160, ff. 373r-373v).

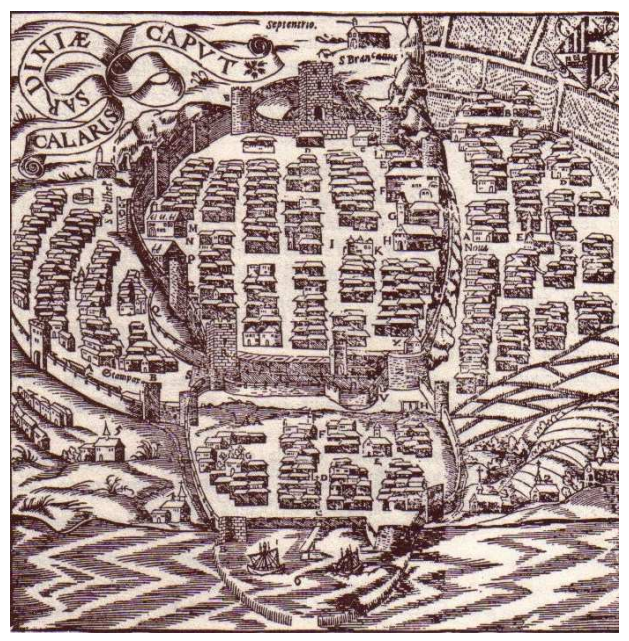

Fig. 2. Mappa di Cagliari di Sigismondo Asquer nella Cosmographia universalis di S. Munster, del 1550.

Sbarcato in Sardegna e prestato giuramento al principio del 1557, il viceré effettuò una visita delle fortificazioni cittadine (Fig. 4): Alghero, Cagliari e, probabilmente, Castellaragonese (ASC, AAR, Parlamenti, vol. 160, f. 41r). Infatti, su queste Città era quasi esclusivamente concentrato il focus della difesa, oltre che su alcuni tratti costieri a forte vocazione produttiva, per la pesca del tonno o la raccolta del corallo, lasciando l'onere della difesa agli stessi operatori (Serreli, 2008a, pp. 121-131). La strategia difensiva fino a questo momento seguita appare essere ancora quella del XIV secolo: per mantenere il Regno sarebbe stato sufficiente difendere a oltranza almeno le Città Regie di Cagliari e Alghero (Casula, 1990). Non veniva neppure presa in considerazione la formazione di una squadra di galere per la difesa mobile delle coste, troppo onerosa per la Corona (Mattone, 1993, pp. 477-509; Mele, 2008, pp. 197-207; Cipollone, 2012, pp. 3839).

La seconda fondamentale decisione di Filippo II è del 6 maggio 1558 e riguarda l'indizione di un Parlamento straordinario con lo scopo di raccogliere le risorse per completare la deffençionem et custodiam dicti Regni (Archivo de la Corona de Aragón [ACA], Cancillería [Canc.], Procesos de Cortes, 49, ff. 2r-2v). Queste necessità sono esplicitate nell'atto di convocazione: "[...] necessitatibus tangenti-bus tutelam et defensionem predicti Regni et precipue arcium et civitatum Callaris, Alguerii, Castri Aragonensis [...]" (ACA, Canc., Procesos de Cortes, 49, ff. 1r-1v).

Non bisogna dimenticare che l'esigenza di adeguare le fortificazioni delle Città era stata già posta a partire dai Parlamenti della seconda metà del XV e del principio del XVI secolo, in particolare nella sessione convocata ad hoc da Giovanni Dusay nel 1504 (Oliva, Schena edd., 1998), ma il problema venne affrontato fino ad allora sempre con provvedimenti disorganici e dettati da necessità contingenti (Serreli, 2008b, pp. 209-218).

In questa occasione, però, per coinvolgere fattivamente il Parlamento nel progetto di difesa e per vincerne le prevedibili resistenze per il donativo straordinario, Filippo II ordinò al tesoriere generale lo stanziamento di altri 20.000 ducati d'oro, da sommare all'ammontare del donativo tolte le spese; la somma si doveva destinare esclusivamente al completamento delle fortificazioni già intraprese, alla riparazione di quelle danneggiate e a eventuali nuove opere ritenute necessarie. Considerato lo stato delle finanze della Corona, lo stanziamento è da considerarsi relativamente consistente ed evidenzia la forte peoccupazione del sovrano e un deciso impegno a realizzare quanto necessario.

In realtà, 20 mila ducati possono essere considerati una somma consistente solo se rapportati al donativo periodicamente versato dagli Stamenti sardi, che alla metà del Cinquecento era di 100 mila ducati d'oro da riscuotere in dieci anni. Si noti che, rapportati all'attualità, 20 mila ducati d'oro corrisponde-rebbero a oltre 10 milioni di Euro. La somma stanziata dalla Tesoreria centra- 
le, invece, potrebbe apparire ben poca cosa se la confrontiamo con i 50 mila ducati spesi dal sovrano tra il 1568 e il 1572 per patrocinare l'edizione della Biblia Polyglotta, oppure con le rendite assegnate ai presuli iberici (Spagnoletti, 2018, pp. 142, 291, 146).

Questi primi provvedimenti -la nomina di un fidato militare alla luogotenenza e la convocazione di un Parlamento straordinario con lo scopo di raccogliere le somme necessarie al completamento, pianificazione e realizzazione di una più funzionale difesa del Regno- fanno intuire un più vasto e organico piano di intervento che riguarda tutta l'amministrazione di questo Stato periferico che aveva accumulato ritardi nella sua organizzazione e nelle strutture di governo rispetto agli Stati peninsulari della Corona (Canet Aparisi, 2017, p. 629). Si tratta, quindi, delle prime tappe di un lento ma deciso disegno che, per quanto riguarda gli aspetti militari, si completerà nei decenni successivi con la costruzione di un sistema statico di difesa costiera (Fig. 4) e la costituzione della Reale Amministrazione delle Torri (Ferrante, 2007, pp. 105-154; Ortu, 2017, pp. 10-19); tutto ciò rende tangibile la fondamentale importanza strategica di questo Regno, benchè periferico e de тиy росо provecho, nel contrasto alla dilagante pressione Ottomana soprattutto riguardo alle potenze barbaresche del nord Africa (Anatra, 1984, pp. 475480). Non si vuole attribuire a Filippo II una visione strategica e politica globale, che apparirebbe forse anacronistica (Parker, 1998), ma è altrettanto evidente per quanto riguarda il Regno sardo, soprattutto nello svolgimento e nelle decisioni prese nel Parlamento del 1558, che al sovrano non sfuggisse la necessità di riformare e accentrare le istituzioni regnicole per sottrarle al controllo dei ceti locali, in modo da rendere più efficiente l'apparato militare difensivo e inserire definitivamente questo Stato, periferico ma strategicamente importante, nel vasto complesso della Monarchia iberica (Mattone, 1993, pp. 477-479; Canet Aparisi, 2017, pp. 623-657). L'esigenza di riforma era emersa negli anni precedenti anche dai risultati dell'inchiesta del visitatore Clavero che si svolse proprio in quegli anni e che portò alla elencazione di una lunga serie di capi d'accusa contro il governatore di Cagliari
Geronimo Aragall e contro lo stesso architetto Rocco Capellino per la cattiva gestione delle già scarse risorse dei donativi precedenti destinate all'adeguamento delle fortificazioni cittadine, utilizzate invece per illeciti scopi personali, lasciando il Regno in balia del pericolo incombente di cadere in mano della coalizione franco turca (Cocco, 1991-1992; Anatra, 1984, pp. 450457, 468-471).

Ma, nonostante 1' "ossessione turca" e tutta l'enfasi posta dal viceré nel sottolineare il rischio di perdere il Regno a vantaggio della coalizione franco ottomana, anche per tentare di coinvolgere i tre Bracci e, soprattutto, le consorterie locali, il primo Parlamento sardo dell'era di Filippo II, si prolungò per oltre due anni, costellato dai ripetuti richiami regi e viceregi alla lealtà e alla celerità. Dal canto loro i rappresentanti del Parlamento giustificavano la ferma opposizione con la povertà delle genti sarde, con la straordinarietà della convocazione (ASC, AAR, Parlamenti, vol. 160, ff. 184r-185v) ma, soprattutto, con l'affievolirsi del pericolo di conquista franco-turca, in considerazione della pace di Cateau Cambrésis del 2 e 3 aprile 1559 (ASC, AAR, Parlamenti, vol. 160, ff. 329r-329v). Tutto ciò fece sì che le aspettative regie per una immediata conclusione dei lavori e per un donativo straordinario, sia come tempistica che come ammontare, venissero del tutto disattese: il donativo verrà approvato solo nell'ottobre del 1560 , nella misura consueta e al termine della riscossione decennale stabilita dal precedente Parlamento del 1553. Il prelievo non venne aumentato rispetto all'ammontare delle sedute precedenti: si trattava di 100000 ducati d'oro di cui 60000 vincolati alla difesa del Regno, futuris necessitatibus in fortificatione, defensione (ASC, AAR, Parlamenti, vol. 161, f. 455r, ff. 649r-649v), come proposto in apertura.

Nonostante l'accordo, travagliato e tardivo, non si ebbe più notizia dei 20000 ducati d'oro stanziati dalla Tesoreria generale nel maggio del 1558, i quali avrebbero dovuto sommarsi ai 60000 ducati previsti dal donativo per le spese militari; più tardi, nel marzo del 1567, Filippo II comunicò di aver disposto che questo stanziamento fosse destinato ad altre necessità. 


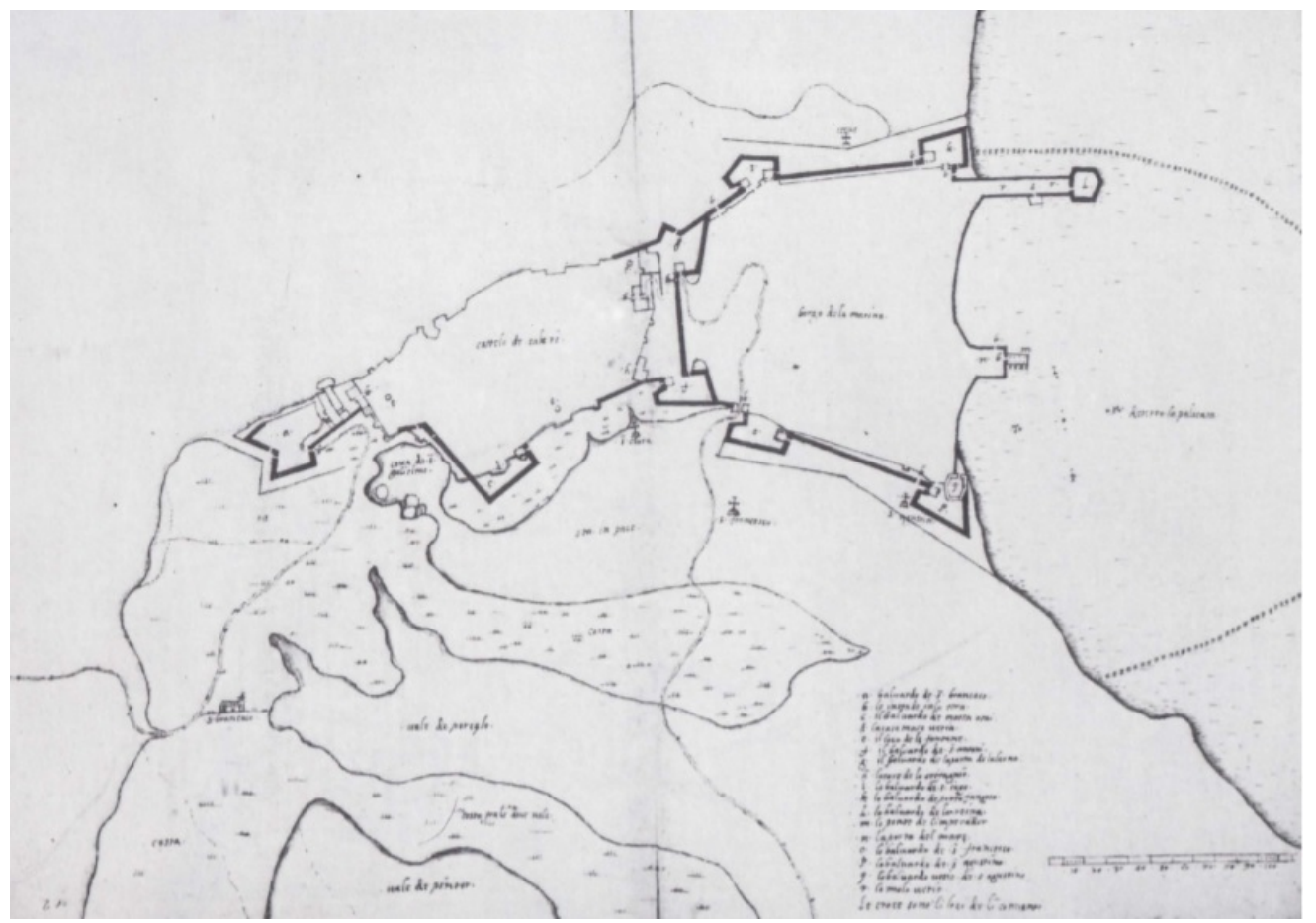

Fig. 3. Fortificazioni di Rocco Capellino nel Castel di Cagliari (Principe, 1988, p. 73, fig. 38).

Questa conclusione -un donativo rimandato di cinque anni e non rispondente alle aspettative regie, i 20000 ducati destinati ad altri disegni e altre emergenze- non favoriva, ovviamente, il progettato completamento delle fortificazioni; perciò, la causa esclusiva della convocazione di questa sessione parlamentare restava non risolta e solo nei decenni successivi verranno trovate faticosamente e per "via extraparlamentare" (Ortu, 2017, p. XV) le risorse finanziarie per completare le fortificazioni delle piazzeforti e realizzare un sistema statico di difesa costiera (Fig. 4).

Nell'immediato, però, il problema restava irrisolto e ciò emerge anche da un primo sguardo alle richieste presentate dalle varie articolazioni dell'Assemblea parlamentare al sovrano, in cambio del donativo: l'esigenza di fortificare e rendere più sicura l'isola compare chiara nelle richieste dei singoli Stamenti e in quella dei tre Bracci riuniti, che verranno valutate dal Consejo e dal sovrano negli anni successivi. Mentre lo Stamento Regio si concentra ovviamente sull'ottimizzazione delle difese cittadine, lo Stamento Militare e quello Ecclesiastico posero invece l'attenzione sulla difesa costiera di tutto il territorio (ASC, AAR, Parlamenti, vol. 161, ff. 471v-610v).

Non era la prima volta che veniva evidenziata la necessità di un sistema di difesa costiero non limitato alle sole Città Regie; nel Parlamento del 1558, però, simile istanza venne proposta, anche se timidamente, dal Braccio Ecclesiastico e da quello Militare (ASCa, AAR, Parlamenti, vol. 161 , f. 544r), in linea con l'orientamento che già si andava sviluppando negli altri Stati mediterranei della Monarchia (Mattone, 2003, pp. 163164; Cipollone, 2012, pp. 47, 56-57).

Finalmente, anche per il Regno sardo si gettavano le basi per pensare a un disegno organico di difesa, con una visione omogenea che non prevedesse solo la fortificazione delle Città Regie, con sporadici provvedimenti dettati dalle contingenze locali, ma che pianificasse la difesa di tutto il perimetro costiero dell'isola; in questo senso, i provvedimenti presi nei decenni successivi per la creazione di una rete di difesa statica, con la costruzione di un sistema di torri lungo le co- 
ste dell'isola e l'istituzione della Reale Amministrazione delle Torri (Mattone, 2003, pp. 160171; Ortu, 2017, pp. 15-17), non possono essere compresi a fondo senza prendere in considera- zione la convocazione, lo svolgimento, le richieste e le decisioni prese durante il Parlamento Madrigal.

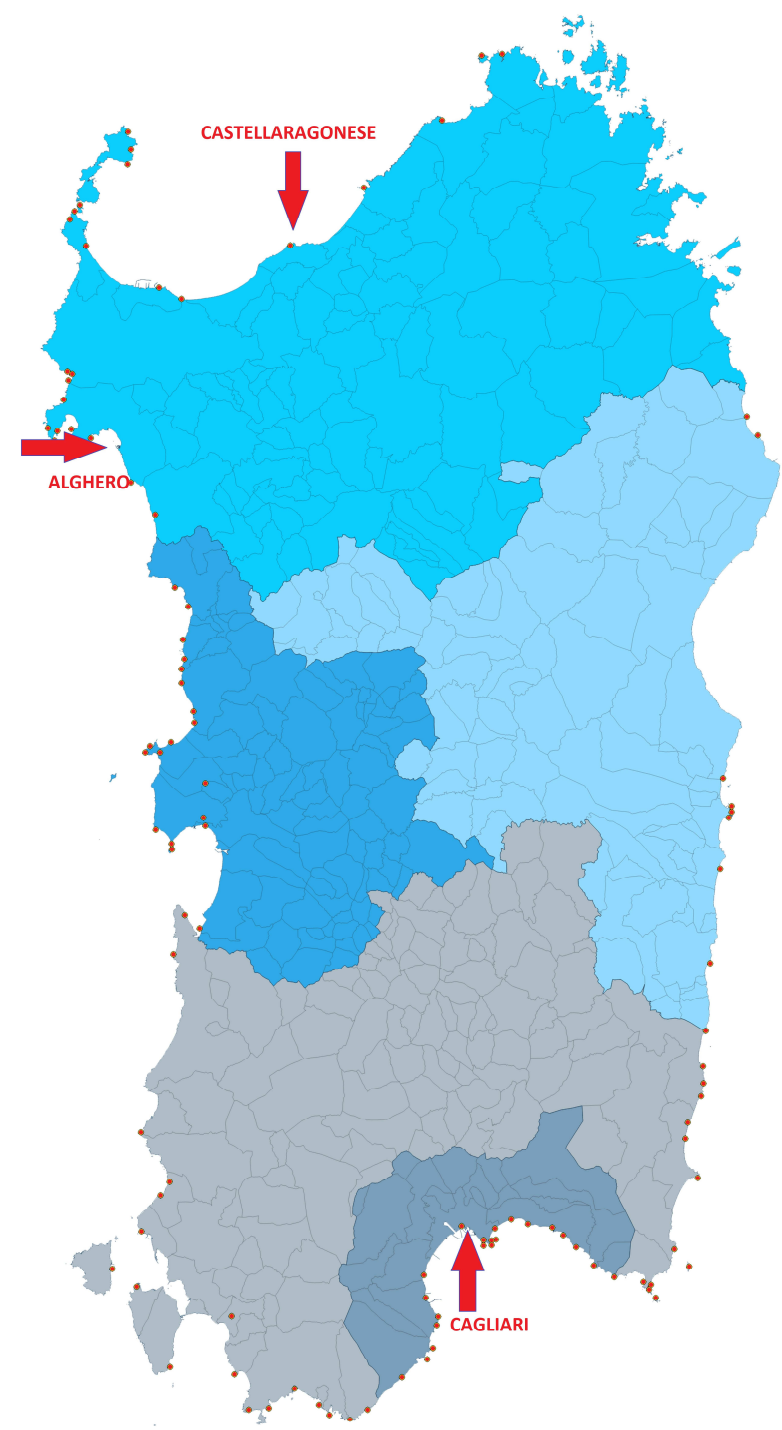

Fig. 4. Principali piazzeforti del Regno di Sardegna e circuito di torri costiere realizzato fra la fine del XVI e il XVII secolo (carta georeferenziata realizzata dall'Ing. Luigi Serra, ISEM CNR).

\section{Bibliography}

Anatra, B. (1984). "Dall'unificazione aragonese ai Savoia", in Day, J.; Anatra, B.; Scaraffia, L. eds. La Sardegna medievale e moderna, in Galasso, G. ed., Storia d'Italia, vol. X, UTET Ed., Torino, pp. 189-663. 
Anatra, B.; Mele, M.G.; Murgia, G.; Serreli, G. edds. (2008). «Contra Moros y Turcos». Politiche e sistemi di difesa degli Stati mediterranei della Corona di Spagna in Età Moderna, 2 vols., Istituto di Storia dell'Europa Mediterranea Ed., Cagliari.

Buyreu, J. (1999). "De Carlos V a Felipe II. La problemática de las abdicaciones y la cuestión virreinal en la Corona de Aragón”, in Belenguer Cebrià, E. coord., Felipe II y el Mediterráneo, Sociedad Estatal para la Commemoración de los Centenarios de Felipe II y Carlos V Ed., Madrid, vol. III, pp. 339-358.

Buyreu, J. (2000). La Corona de Aragón de Carlos V a Felipe II. Las instrucciones a los virreyes bajo la regencia de la princesa Juana (1554-1559), Sociedad Estatal para la Commemoración de los Centenarios de Felipe II y Carlos V Ed., Madrid.

Cancila, R. ed. (2007). "Mediterraneo in armi (secc. XV-XVIII)", in Quaderni-Mediterranea: ricerche storiche, 4, NDF Ed., Palermo.

Canet Aparisi, T. (2017). "La creación de la Real Audiencia de Cerdeña (1562-1573): un periodo decisivo para el gobierno del reino y su integración en el sistema administrativo hispánico”, in Arrieta Alberdi, J., Gil, X., Morales Arrizabalaga, J. edds., La diadema del Rey: Vizcaya, Navarra, Aragón y Cerdeña en la Monarquía de España (siglos XVI-XVIII), Universidad del País Vasco Ed., s.1., pp. 623-657.

Casula, F.C. (1990). La Sardegna aragonese, Chiarella Ed., Sassari.

Cipollone, V. (2012). "La politica militare di Filippo II sul fronte mediterraneo", in Mele, G. ed., Tra Italia e Spagna. Studi e ricerche in onore di Francesco Manconi, CUEC Ed., Cagliari, pp. 35-58.

Cocco, M.M. (1991-92). "Fatti e misfatti nella Sardegna del '500. Relazione Clavero-La Maison (1558-1561)”, Annali della Facoltà di Magistero dell'Università di Cagliari, 15, 2, pp. 3-82.

Elliot, J.H. (2002). Imperial Spain 1469-1716, Penguin Books Ed., London.

Ferrante, C. ed. (2007). "Le istituzioni militari del Regnum Sardiniae nei secoli XVI-XVIII: fonti e percorsi di ricerca nell' Archivio di Stato di Cagliari”, Quaderni Bolotanesi, 33, pp. 105-154.

Manconi, F. (2010). La Sardegna al tempo degli Asburgo. Secoli XVI-XVII, Il Maestrale Ed., Nuoro.

Mattone, A. (1993). "L'amministrazione delle galere nella Sardegna spagnola”, in D'Arienzo, L. ed., Sardegna, Mediterraneo e Atlantico tra Medioevo ed età Moderna, Bulzoni Ed., Roma, pp. 477-509.

Mattone, A. (2003). "Il Regno di Sardegna e il Mediterraneo nell'età di Filippo II. Difesa del territorio e accentramento statale", in Filippo II e il Mediterraneo, Laterza Ed., Roma-Bari, pp. 147-264.

Mattone, A. (2007). "Castellaragonese. Una città-fortezza nel Mediterraneo moderno (XVI-XVIII secolo)", in Mattone, A.; Soddu, A. edd., Castelsardo. Novecento anni di storia, Carocci Ed., Roma, pp. 459-539.

Mele, G. (2008). "Torri o galere? Il problema della difesa costiera in Sardegna tra XVI e XVIII secolo", in "Contra Moros y Turcos». Politiche e sistemi di difesa degli Stati mediterranei della Corona di Spagna in Età Moderna, vol. I, Istituto di Storia dell'Europa Mediterranea Ed., Cagliari, pp. 197-207.

Mele, G. (2015). "Il Regno di Sardegna come realtà di frontiera nel Mediterraneo del secolo XVI: un progetto di conquista franco-turca della metà del Cinquecento", in Guia Marín, L.J.; Mele, M.G.R.; Tore, G., Identità e frontiere. Politica, economia e società nel Mediterraneo (sec. XIV-XVIII), Franco Angeli Ed., Milano, pp. 135-146.

Mele, G. (2017). La rivoluzione militare in Sardegna: fortificazioni, presidi e milizia terriotoriale. Fonti d'archivio (1553-1611), Universitas Studiorum Casa Editrice Ed., Mantova.

Murgia, G. (2012). "Paura corsara e problemi di difesa tra Cinque e Seicento", in Murgia, G. ed., Un'isola, la sua storia. La Sardegna tra Aragona e Spagna (secoli XIV-XVII), Grafica del Parteolla Ed., Dolianova, pp. 61-119.

Nocco, S. (2009). "I progetti per le fortificazioni nella Sardegna moderna", RiMe (Rivista dell'Istituto di storia dell'Europa Mediterranea), 2, pp. 131-141.

Oliva, A.M.; Schena, O. edd. (1998). I Parlamenti dei viceré Giovanni Dusay e Ferdinando Giròn de Rebolledo (1495, 1497, 1500, 1504-1511), Acta Curiarum Regni Sardiniae, 5, Consiglio Regionale della Sardegna Ed., Cagliari.

Oliva, A.M. (2005). "Il Consiglio Regio nel Regno di Sardegna. Prime ricerche", in Ferrer i Mallol, M.T.; Mutgé i Vives, J.; Sánchez Martínez, M. edd., La Corona catalanoaragonesa i el seu entorn mediterrani a la baixa edat mitjana, CSIC - Instituciò Milà i Fontanals - Departament d'Estudis Medievals Ed., Barcelona, pp. 205-238. 
Ortu, G.G. (2017). "Preface-Prefazione", in Gannattasio, C.; Grillo, S.M.; Murru, S., Il sistema di torri costiere in Sardegna. Storia della tecnica edilizia e restauro dei monumenti, L'ERMA di BRETSCHNEIDER Ed., Roma, pp. X-XIX.

Parker, G. (1998). La gran estrategia de Felipe II, Alianza Ed., Madrid.

Principe, I. (1988). Cagliari. Le città nella storia d'Italia, Laterza Ed., Roma-Bari.

Serreli, G. (2007). "Sardinya Kralligi'nin savunma sistemleri ve Terranova'nin Turgut Reis tarafindan yagmalanmasi”, in Türkler ve Deniz, Kitap Yayinevi Ed., Istambul, pp. 175-185.

Serreli, G. (2008a). "Le opere di difesa delle attività produttive nel Regno di Sardegna nel XVI secolo. Il caso di Capo Carbonara”, RiMe (Rivista dell'Istituto di storia dell'Europa Mediterranea), 1, pp. 121-131.

Serreli, G. (2008b). “... Fabricar en su continente torres y bastiones. I problemi dell'organizzazione difensiva nel Regno di Sardegna nella prima metà del XVI secolo", in «Contra Moros y Turcos». Politiche e sistemi di difesa degli Stati mediterranei della Corona di Spagna in Età Moderna, vol. I, Istituto di Storia dell'Europa Mediterranea Ed., Cagliari, pp. 209-218.

Spagnoletti, A. (2018). Filippo II, Salerno Ed., Roma. 